\title{
The Embodiment of a DUAL/AMBR Based Cognitive Model in the RASCALLI Multi-Agent Platform
}

\author{
Stefan Kostadinov and Maurice Grinberg \\ 1 Central and Eastern European Center for Cognitive Science, \\ New Bulgarian University, 21, Montevideo St., Sofia 1618 , Bulgaria \\ stefan@yobul.com \\ 2 mgrinberg@nbu.bg
}

\begin{abstract}
The current paper explores some of the cognitive abilities of an embodied agent based on the DUAL/AMBR architecture. The model has been extended with several capabilities like continuous operation and learning based on encoding of episodes and general knowledge. A new mechanism of analogical transfer has been implemented and demonstrated on a simulated interaction with a user. A focus of interest discussed throughout the paper is how a cognitive model can be embodied in a virtual environment and what are the benefits of combining soft cognitive capabilites with hard AI based platform. The latter is based on a Mind-Body-Environment metaphor which positions the cognitive agent in a situation similar to the one of a robot in a real environment. In the paper, results from simulations of simple interactions with a hypothetical user are presented and the internal cognitive mechanisms are discussed.
\end{abstract}

\section{Introduction}

DUAL is a cognitive architecture based on micro-agents which have a hybrid connectionist-symbolic nature. AMBR (Associative Memory Based Reasoning) is a model of analogy making based on DUAL [1]. The DUAL/AMBR architecture is based on the principle of local, context sensitive, and emergent parallel computation and integration and interaction of memory retrieval, mapping, transfer, and evaluation in reasoning by analogy. Work reported in recent publications (see [5] and [?]) has implemented several new mechanisms, including anticipatory ones, which turned the model into a fully operational cognitive module integrating memory, reasoning, perception, decision making and action. This is the only model which integrates explicitly anticipation based on analogy and in which the anticipatory mechanisms are used essentially in perception, evaluation and action [5].

The RASCALLI platform is a modular environment for modeling and implementation of artificial multi-agent systems giving the possibility to incorporate cognitive models [2]. This paper, based on a simulated interaction episode, explores how a cognitive model can become an integrated part of the platform 
and how it can augment the functionality of otherwise powerful computational mechanisms in the context of a constant interaction with a user. The expectation is that such models will bring the flexibility, context sensitivity, and adaptability (via perception and learning) characteristic of human behaviour. On the the other hand the integration of cognitive components will lead to higher believability, individuality (human-likeness), and specialization to a specific user and the related higher usability and effectiveness of the interaction and task completion (e.g. information retrieval).

The use of Mind-Body-Environment metaphor has been proposed in [6]. The idea is to abstract the use of a cognitive model from the specific application, Embodied Conversational Agent (ECA) or robot, and describe the interactions of the cognitive part with the other parts of the system in terms of a Mind (perception, memory, representations etc.), Body (sensors, effectors, and more generally Tools) and Environment.

The contributions of this paper are in the attempt to build a cognitive module which can be used in the same way in robots and embodied agents and in the specific development of new mechanisms which allow processes from perception to action to take place based on analogy-making. On the other hand the cognitive module encodes personalized information about specific past episodes and not only general knowledge. The latter is demonstrated in a scenario which simulates an interaction with a user and question answering based on analogy reasoning.

\section{The RASCALLI Architecture}

The RASCALLI platform provides a modular and flexible development environment for modeling and realization of artificial systems that can be endowed with various cognitive abilities [2]. The architecture is component-based and allows researchers to choose from deployed components in order to make a new realization of RASCALLI. This approach benefits distributed development by allowing independent development of components. The components can communicate with each other in the terms of a global, shared Ontology. Each component must define its input and output capabilities to the global Ontology, thus making itself available to the other components.

The RASCALLI platform allows creation of different architectures and the specific one being described is inspired by the 'Mind-Body-Environment' metaphor. It features the Mind, with its specific knowledge structures and mechanisms, the Sensory-Motor Layer, making a mediated connection of the Mind, and the Tools of RASCALLI - the sensors and effectors that work with the SensoryMotor Layer and perceive or act on the Environment. The Body, along with the Sensory-Motor Layer provides represented knowledge to the Mind and thus makes mediated connection between the mind and the world. [6]

The DUAL/AMBR cognitive module is integrated in the RASCALLI platform as a Mind component which can use different Tools (sensors and effectors). In the example, presented below such Tools are the Input Processing (IP) and 
Multi-Media Generation (MMG) tools, allowing communication with the user of the system(see [2] for details).

The processing of the input by the user is made with state-of-the-art NLP methods, developed especially for the RASCALLI project. The resulting processing is made with methods that cannot only localize relation arguments but also assign their exact target argument roles [7].

The ECA that the user interacts with is a full-size 3D animation, that demonstrates lips synchronization when it talks and various effects that make it realistic. [8] See Picture 1 on page 3

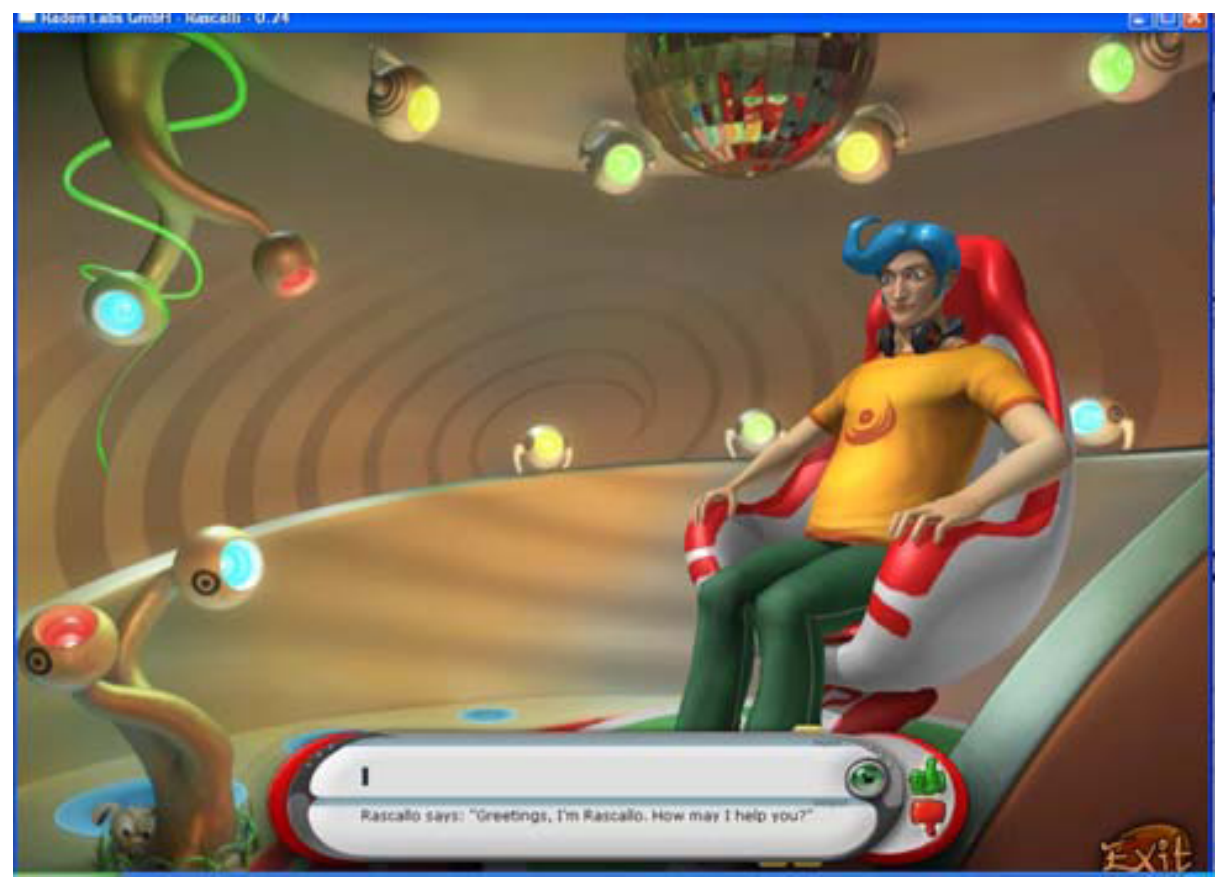

Fig. 1. The initial RASCALLI ECA. Female version available. [2]

\section{The DUAL/AMBR based Mind of RASCALLI}

The DUAL cognitive architecture ([1]) is hybrid and combines symbolic with connectionist approaches, by integrating them at the micro-level. It is based on emergent computations so that the global behavior of the architecture emerges from local interactions among a multitude of micro-agents. The behavior of DUAL changes continuously in response to the influence of the changes in context based on a activation spreading mechanism. 
The architecture has a Long-Term Memory (LTM) and a Working Memory (WM) which consists of the input and goal and the most active part of LTM. The input and goal of the system are the sources of activation thus activating the most relevant part of LTM. The latter is then involved in symbolic operations, like marker passing, correspondence hypotheses rating, etc. (see [5] for details), which support the reasoning processes.

AMBR is a model for analogy making based on DUAL. It treats analogy making as an emergent result of the common work of several overlapping subprocesses perception, retrieval, mapping, transfer, evaluation, and learning. AMBR is based on the mechanism of mapping similar or analogical parts in WM, creating hypotheses for correspondences (typically between input and goal and episodes from LTM). The final mapping is established based on a constraint satisfaction network which discards inconsistent hypotheses. The basic mechanisms used in AMBR include spreading activation, marker passing, structural correspondence, constraint satisfaction, rating and promotion of correspondence hypotheses.

The development of the DUAL/AMBR model in the robotic context [5] added new anticipatory mechanisms to the the mechanisms mentioned above. Anticipated objects and relations from LTM episodes are added to the problem at hand as expectations. Anticipatory mechanisms are used also for active perception and selective attention [5]. In this paper, we present a new mechanism used for transfer and evaluation of knowledge between distant domains which will be discussed bellow.

\section{Knowledge Representations}

Each DUAL/AMBR based model consists of a set DUAL agents with various types and with various properties [3]. These agents can be concept agents, representing classes of homogeneous entities organized in a semantic network. They can also be instances of classes (instance agents) building episodes, hypothesis agents (representing possible correspondences between agents), cause agents (representing causal relations), and action agents (coding procedural knowledge). A special class of agents are the anticipation agents which stand for expected entities or relations.

As stated in section 1 , the cognitive module has only a mediated perception of the world via the sensor (Tools) which provide RDF messages, representing events or action commands. All messages exchanged between the Mind and the Tools are based on a shared, global ontology [2]. Additionally to the global shared ontology, the agent LTM has a specific knowledge needed to organize its interactions with the environment into memory episodes, actions, causal relations, etc.

Knowledge base. The knowledge base contains information about the RASCALLI, the Environment, the Tools and the actions related to them, causal 
relations between actions and outcomes etc. It is built mostly of concepts, including concepts of relations but there are also specific propositions and specific knowledge.

Instinct coalitions. As the architecture is intended to support different types of (including plug-and-play) tools, the way each of them is used have to be stored in LTM and accessible on demand. The instinct coalitions are examples of tool usage. They contain all required parameters and the message scheme required by the Tools. Those coalitions are linked to the various actions the Mind can trigger and are used as a template every time the Mind decides to act in the Environment. Instinct coalitions are added to LTM on tool registration and removed from LTM when the tool is unregistered.

Episodes. There are four types of episodes used:

1. Knowledge episodes - they contain factual information collected in the past by the agent. Knowledge episodes are created every time any Tool brings new information to the Mind;

2. Usage schemes - describe the various actions that can provide information, e.g. search in a data source. Those episodes can be automatically added to the Mind, especially if the data source can provide the type of information it contains;

3. User satisfaction - provide the need to share the most relevant information found with the user. There are few of those episodes and they form the basis of the RASCALLI's desire to be cooperative and to provide information to the user;

4. Interaction with the user - keeping track of all the life of RASCALLI. Those episodes are added constantly during the lifetime of RASCALLI.

\section{Scenario and DUAL/AMBR Mechanisms}

In a previous paper [6], a "Context scenario" in which a number of questions were asked to the RASCALLI and interesting context effects have been demonstrated. The simulation showed the ability of the system to preserve context between utterances.

\section{Reasoning and transfer mechanisms}

The user is supposed to communicate with the agent, by typing the utterances in an input box and the answers are given back vocally by the agent ([2]). The analysis of the utterance provides such information as the type of entities in the semantic representation, the focus of question (what is asked for), etc. ([7]).

The utterances are expressed as semantic graph by the IP Tool and translated in frame-based format by the Sensory-Motor Layer before being sent to the Mind. 
After that the Mind starts working in the context of its current state and the task. Each of the instances from the current context starts to spread activation in LTM. The most active elements from WM establish correspondence hypotheses which some of which becom mappings and lead to the transfer from WM to the task at hand (represented as a target episode). If there is no specific a specific answer found, actions that could provide it are transferred and executed. If a search is made, the agent retains the information that it gets through the Tools in knowledge episodes so that it can provide this information on demand in the future $([6])$.

\section{Complementary transfer and evaluation mechanism}

The newly developed mechanism of complementary transfer and evaluation allows the Mind to combine pieces of information from different domains in order to solve a task. The general algorithm makes mapped consequences to transfer their reasons (a causal relation has reason and a consequence), and if the reasons are distant from the target domain, to find the closest matches within the domain of the question. They are found from the general or experiential knowledge via taxonomy-propagating intersections. This is demonstrated in the following scenario.

The test utterance is "Tell me a famous musician.".

The internal knowledge is set up in such a way that there is no information about musicians being famous. Actually, the famous concept is not currently part of the musical domain and there is no information on how "famous" relates to "singers". There are however, several episodes that are related and enter the WM:

- Information about artists and Grammy awards can be obtained from data source "X";

- Musicians are awarded with Grammy;

- Artists are awarded with Oscar;

- Actor "Y" is famous because of winning an Oscar.

The latter episode is in a different domain than the requested entity, so it cannot be transferred directly. Although the domain of music artists is not so different than the domain of movie artists, it is distant enough not to allow direct transfer.

The process of complementary transfer goes as follows: first, mappings emerge between the requested musician and musicians and artists from the KB. Another mapping produced is the one linking the famous (musician) relation to the famous (artist) relation in the KB. Then, the causal relation that links the "being famous" to "winning an Oscar" transfers the reason for the mapped relation "winning", but it cannot transfer the argument ("Oscar"), as it is not present in the musical domain. Another mapping however is made to the base episode stating that "Artists are awarded with Oscar", so an instance of "Grammy" is appended to the transferred relation "winning". 
Thus the knowledge that musicians can win Grammy awards is used to anticipate that the musician in question is famous, because winning a Grammy award. The result of those two mappings is the transfer of the "has a Grammy award" information to the target. This transfer does not provide an answer to the user request, but it enlarges the task description with additional information, expected to lead to the desired information. Thus the task that is impossible to fulfill ("find a famous musician") is modigied to "find a musician with a Grammy".

This time, again there is not a single musician with Grammy in the LTM, but there is an episode, stating that there is a data source that contains information on Grammy awards and musicians. From here on, the system activates the knowledge that there is information about musicians winning Grammy in a specific data source, and this data source is asked to provide such a musician.

\section{Discussion and Conclusion}

The results from the simulated conversations demonstrate some of the functionalities of the ECA specifically related to the cognitive model implementing the Mind. The behaviour of the agent is mainly due to the episodic part of LTM which is unique to an agent and its user and encode individual experiential information different from the available knowledge in ontologies and databases. This 'personal' part of Rascalli memory is a crucial advantage of the cognitive model involved.

It is expected that the long experience of the interaction between the user and the agent and the agent and the environment will lead to the formation of complex structures of episodes and general knowledge which will make analogy making mechanisms very efficient and unique.

\section{Acknowledgments}

The current research and development project has been partly financed by the FP6 EU project RASCALLI.

\section{References}

1. Kokinov B. \& Petrov A. (2000): Integration of Memory and Reasoning in AnalogyMaking: The AMBR Model. In: Gentner, D., Holyoak, K., Kokinov, B. (eds.) The Analogical Mind: Perspectives from Cognitive Science, Cambridge, MA: MIT Press. 2. Brigitte Krenn. (2008): RASCALLI. Responsive Artificial Situated Cognitive Agents Living and Learning on the Internet. In Proceedings of the International Conference on Cognitive Systems (CogSys 2008), University of Karlsruhe, Karlsruhe, Germany, April 2 - 4, 2008.

3. Kokinov B. (1994): The DUAL Cognitive Architecture. A Hybrid Multi-Agent Approach. In A. Cohn (ed.) Proceedings of the Eleventh European Conference of Artificial Intelligence. London: John Wiley \& Sons, Ltd, pp. 203-207. 
4. Pattie Maes (1987): Agents that Reduce Work and Information Overload in Communications of the ACM, July 1987, Vol.37, No. 7, pp.30-40

5. Petkov G., Naydenov Ch., Grinberg M., Kokinov B. (2007): Building Robots with Analogy-Based Anticipation. In: C. Freksa, M. Kohlhase, and K. Schill (eds.) Proceedings of the 29th German Conference on Artificial Intelligence (KI-2006), LNAI 4314, Bremen, Germany, 2007. Springer, pp. 72-86.

6. Kostadinov S., Petkov G., Grinberg M. (2008): Embodied conversational agent based on the DUAL cognitive architecture. In proceedings of WEBIST 2008 International Conference on Web Information Systems and Technologies.

7. Feiyu Xu, Hans Uszkoreit, Hong Li. (2008): A Seed-driven Bottom-up Machine Learning Framework for Extracting Relations of Various Complexity. In Proceedings of ACL 2007, Prague.

8. Nebula platform (2007) http://www.radonlabs.de/technologynebula2.html

9. MARY text-to-speech engine (2008) http://mary.dfki.de 